\title{
Automatic carrier phase delay synchronization of PGC demodulation algorithm in fiber-optic interferometric sensors
}

\author{
Changbo Hou ${ }^{1,2}$ and Shuai Guo ${ }^{{ }^{*}}$ \\ ${ }^{1}$ College of Information and Communication Engineering, \\ Harbin Engineering University, Harbin, 150001, China. \\ [e-mail: guoshuai_lemonade@163.com] \\ ${ }^{2}$ College of Physics and Optoelectronic Engineering, \\ Harbin Engineering University, Harbin, 150001, China. \\ [e-mail: houchangbo@hrbeu.edu.cn] \\ *Corresponding author: Shuai Guo
}

\begin{abstract}
Phase-generated carrier (PGC) demodulation algorithm is the main demodulation methods in Fiber-optic interferometric sensors (FOISs). The conventional PGC demodulation algorithms are influenced by the carrier phase delay between the interference signal and the carrier signal. In this paper, an automatic carrier phase delay synchronization (CPDS) algorithm based on orthogonal phase-locked technique is proposed. The proposed algorithm can calculate the carrier phase delay value. Then the carrier phase delay can be compensated by adjusting the initial phase of the fundamental carrier and the second-harmonic carrier. The simulation results demonstrate the influence of the carrier phase delay on the demodulation performance. PGC-Arctan demodulation system based on CPDS algorithm is implemented on SoC. The experimental results show that the proposed algorithm is able to obtain and eliminate the carrier phase delay. In comparison to the conventional demodulation algorithm, the signal-to-noise and distortion ratio (SINAD) of the proposed algorithm increases 55.99dB.
\end{abstract}

Keywords: Phase-generated carrier, fiber-optic interferometric sensors, carrier phase delay synchronization, orthogonal phase-locked 


\section{Introduction}

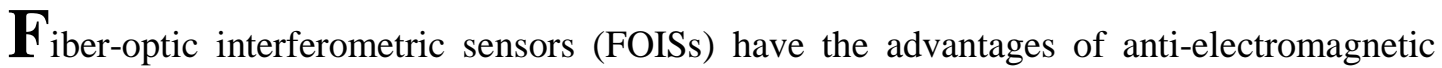
interference, good linearity, wide frequency response range and large dynamic range [1-4]. The main problem of FOISs is to design a demodulation system with large dynamic range. In recent years, there are some demodulation methods which use machine learning $[5,6]$. However, phase-generated carrier (PGC) demodulation algorithm has the advantages of wide dynamic range, high linearity and high sensitivity. It has been widely used in FOISs [7-11]. The conventional PGC demodulation algorithms are the differential-and-cross-multiplying (PGC-DCM) [12, 13] and the arctangent (PGC-Arctan) [14, 15]. These demodulation methods make the communication performance of FOISs system much better than that of traditional communication [16].

However, a phase delay between the interference signal and the carrier signal, due to the time delay caused by the laser transmission delay, the electric transmission on electronic processing circuit, the conversion time of the analog-to-digital converter (ADC), the photoelectric conversion and external environment noise [17]. The carrier phase delay will cause harmonic distortion in the demodulation results [18-20]. Moreover, for the same time delay, the carrier phase delay will rise with the increase of carrier modulation frequency [21].

In order to eliminate the effect of carrier phase delay, several methods have been proposed. S. C. Huang et al. proposed a improved PGC-DCM demodulator compensated for the transmission delay of the optical fiber [22]. Besides, the demodulator adopted the fundamental and the second-harmonic square wave signal to compensate the carrier phase. S. H. Zhang et al. proposed a method which introduce a compensating phase to the carrier. The carrier synchronize with the interference signal when the output of the low pass filter is maximum. The real-time carrier phase delay compensation can be realized by the algorithm [21]. S. W. Li et al. proposed an modified digital PGC demodulation algorithm. The method eliminates the carrier phase delay effect by the synchronous carrier restoration (SCR) method [23]. The method directly extract frequency and phase of the carrier signal from the interference signal. And the carrier signal can be synchronized by the information. Aleksandr N. Nikitenko et al. proposed a method which get orthogonal and in-phase components of the interference signal by using sine and cosine signals. The method can realize the carrier phase delay compensation in real time to mitigate the carrier phase delay effect without any additional phase adjusting. [19]. J. D. Xie et al. proposed a method to obtain carrier phase delay by robust extraction. The method uses the first, second, and fourth order orthogonal harmonic components to eliminate nonlinear error of PGC demodulation algorithm [24].

In this paper, a novel carrier phase delay synchronization algorithm for all-digital PGC demodulation based on SOC is proposed. This method is suitable for PGC-DCM and PGC-Arctan methods to compensate the phase delay in real time. It uses a pair of in-phase and quadrature carrier signals to calculate the carrier phase delay. Then the carrier phase delay can be adjusted to be zero. In section 2, the effect of carrier phase delay in PGC demodulation algorithm. And the principle of automatic carrier phase delay synchronization (CPDS) algorithm are analyzed. In section 3, the simulation present the effectiveness of CPDS algorithm. In section 4, the SoC platform which include FPGA and ARM is shown. And the experiments to show the practicability of the CPDS algorithm are given. 


\section{Theory}

\subsection{The Principle of PGC Demodulation Algorithm}

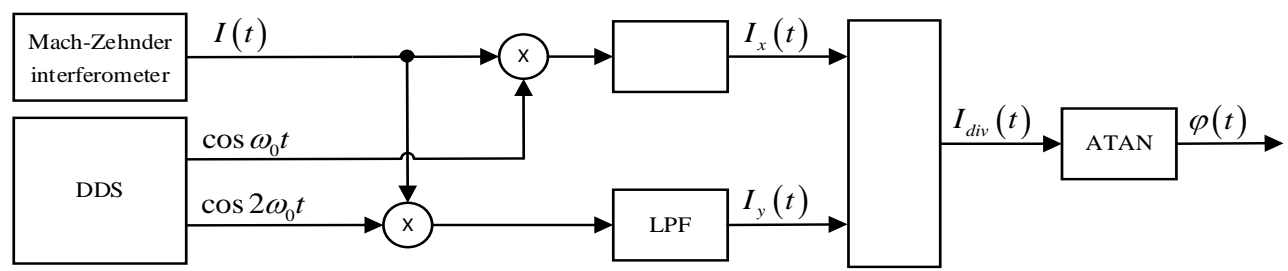

Fig. 1. Principle of PGC-Arctan demodulation algorithm.

The interference signal [25-28] can be described as

$$
I(t)=A+B \cos \left[C \cos \omega_{0} t+\varphi(t)\right]
$$

In Eq (1), $A$ is the DC offset, $B$ is the AC amplitude. $C$ is the phase modulation depth. $\omega_{0}$ is the carrier frequency. $\varphi(t)$ is the sum of the phase signal to be demodulated and the noise. The principle of PGC demodulation algorithm is shown in Fig. 1. DDS is direct digital frequency synthesizer. LPF is the low-pass filter. DIV is the division. ATAN is arctangent operation. MUL is the multiplier. The signal sended from the Mach-Zehnder interferometer multiplies with the fundamental and the second-harmonic of carrier modulation signal. After expanding to a Bessel function can obtain

$$
\begin{array}{rl}
I(t) \times \cos \omega_{0} t \\
= & A \cos \omega_{0} t+B\left(J_{0}(C)+2 \sum_{k=1}^{\infty}(-1)^{k} J_{2 k}(C) \cos 2 k \omega_{0} t\right) \cos \omega_{0} t \cos \varphi(t) \\
& -2 B\left(\sum_{k=0}^{\infty}(-1)^{k} J_{2 k+1}(C) \cos (2 k+1) \omega_{0} t\right) \cos \omega_{0} t \sin \varphi(t) \\
I(t) \times \cos 2 \omega_{0} t & A \cos 2 \omega_{0} t+B\left(J_{0}(C)+2 \sum_{k=1}^{\infty}(-1)^{k} J_{2 k}(C) \cos 2 k \omega_{0} t\right) \cos 2 \omega_{0} t \cos \varphi(t) \\
= & 2 B\left(\sum_{k=0}^{\infty}(-1)^{k} J_{2 k+1}(C) \cos (2 k+1) \omega_{0} t\right) \cos 2 \omega_{0} t \sin \varphi(t)
\end{array}
$$

Where $J_{k}(C)$ is the $k$-order Bessel function. Then the signals get into the low-pass filters. A pair of non-strict quadrature components $I_{x}(t)$ and $I_{y}(t)$ are obtained

$$
\begin{array}{r}
I_{x}(t)=-B J_{1}(C) \sin \varphi(t) \\
I_{y}(t)=-B J_{2}(C) \cos \varphi(t)
\end{array}
$$


Where $J_{1}(C)$ is the first-order Bessel function. $J_{2}(C)$ is the second-order Bessel function. Next the output signals of the low-pass filters can be dealt with PGC-Arctan or PGC-DCM demodulation method to get the sensing phase signal.

For the PGC-Arctan demodulation algorithm, after dividing $I_{x}(t)$ and $I_{y}(t)$ yields

$$
I_{d i v}(t)=\frac{J_{1}(C)}{J_{2}(C)} \tan \varphi(t)
$$

In Eq (6), $C$ is adjusted to 2.63 rad to make $J_{1}(C)=J_{2}(C)$, So that the effect of Bessel function on the demodulation results can be minimized,. Eq (6) can be rewritten as

$$
I_{d i v}(t)=\tan \varphi(t)
$$

After the operation of arctangent, the sensing phase signal $\varphi(t)$ can be got.

For the PGC-DCM demodulation algorithm, the signal through the operation of differential-and-cross- multiplying. And the results are described as

$$
\begin{gathered}
\frac{d I_{x}(t)}{d t} \times I_{y}(t)=B^{2} J_{1}(C) J_{2}(C) \frac{d \varphi(t)}{d t} \cos ^{2} \varphi(t) \\
\frac{d I_{y}(t)}{d t} \times I_{x}(t)=-B^{2} J_{1}(C) J_{2}(C) \frac{d \varphi(t)}{d t} \sin ^{2} \varphi(t)
\end{gathered}
$$

After the signal through the operations of subtracting and integrating. The demodulation result can be expressed as

$$
I_{S U B}=2 B^{2} J_{1}(C) J_{2}(C) \varphi(t)
$$

In Eq (10), $C$ is adjusted to 2.37 rad to make the change rate of $J_{1}(C) J_{2}(C)$ minimum. So that the effect of Bessel function on the demodulation results can be minimized.

\subsection{The Influence of Carrier Phase Delay in PGC Demodulation Algorithm}

In practical applications, due to the laser transmission delay in the fiber-optic interferometric sensor, the conversion time delay of ADC in the photodetection and the circuit board. the carrier phase delay exists between the carrier signal and the interference signal. Considering the phase delay, Eq. (1) should be rewritten as

$$
\begin{aligned}
I(t) & =A+B \cos \left[C \cos \left(\omega_{0}(t+\Delta t)\right)+\varphi(t)\right] \\
& =A+B \cos \left[C \cos \left(\omega_{0} t+\Delta \varphi\right)+\varphi(t)\right]
\end{aligned}
$$

Where $\Delta t$ is the time delay, and $\Delta \varphi$ is the carrier phase delay between the carrier signal and the interference signal. So that Eq. (4) and Eq. (5) should be rewritten as

$$
\begin{gathered}
I_{x}(t)=-B J_{1}(C) \sin \varphi(t) \cos \Delta \varphi \\
I_{y}(t)=-B J_{2}(C) \cos \varphi(t) \cos 2 \Delta \varphi
\end{gathered}
$$

For the PGC-Arctan demodulation algorithm, the demodulation result becomes

$$
\varphi^{\prime}(t)=\arctan \left(\frac{\cos \Delta \varphi}{\cos 2 \Delta \varphi} \tan \varphi(t)\right)
$$


It is obvious that $\varphi(t)$ can not be obtained by the operation of arctangent because of the existence of $\cos \Delta \varphi / \cos 2 \Delta \varphi$.

For the PGC-DCM demodulation algorithm, the demodulation result becomes

$$
I_{\text {SUB }}=B^{2} J_{1}(C) J_{2}(C)(\cos \Delta \varphi \cos 2 \Delta \varphi) \varphi(t)
$$

It is clear that $\cos \Delta \varphi=0$ when $\Delta \varphi=\frac{\pi}{2}+k \pi(k=1,2,3 \ldots)$ which will cause demodulation failure.

\subsection{The Principle of Automatic Carrier Phase Delay Synchronization}

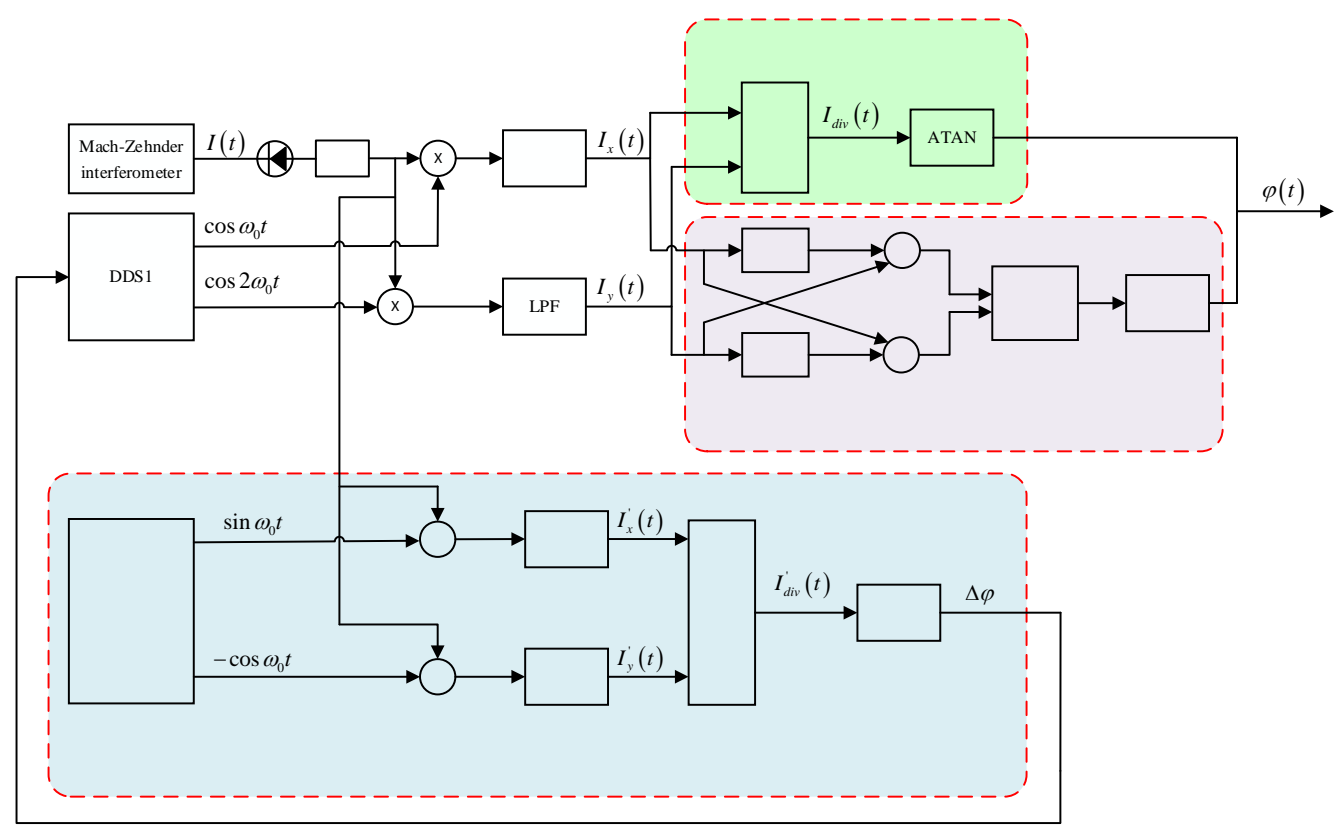

Fig. 2. Principle of PGC demodulation algorithm with the automatic carrier phase delay synchronization.

The PGC demodulation algorithm with the automatic carrier phase delay synchronization is shown in Fig. 2. SUB is subtraction. INT is integral. The photodetector (PD) converts the optical interference signal from the Mach-Zehnder interferometer to electrical signal. Then the electrical interference signal is sampled by the ADC. Last the sampled signal enter the PGC-Arctan and PGC-DCM demodulation module to obtain the sensing phase signal. In order to make up the carrier phase delay $\Delta \varphi$, the carrier phase delay synchronization (CPDS) algorithm based on orthogonal phase-locked is introduced to the PGC demodulation algorithm. $\Delta \varphi$ can be calculated by CPDS module. And CPDS module can adjust the initial phase of the carrier signal and its second harmonics which are generated by DDS. 
CPDS module is shown in Fig. 2. It is necessary to synchronize the carrier phase delay. So CPDS algorithm is introduced to the PGC demodulation algorithm. The interference signal multiplies with the orthogonal and fundamental of carrier signal. After expanding to a Bessel function can obtain

$$
\begin{aligned}
& I(t) \times \sin \omega_{0} t \\
&= A \sin \omega_{0} t+B\left(J_{0}(C)+2 \sum_{k=1}^{\infty}(-1)^{k} J_{2 k}(C) \cos 2 k \omega_{0} t\right) \sin \omega_{0} t \cos \varphi(t) \\
&-2 B\left(\sum_{k=0}^{\infty}(-1)^{k} J_{2 k+1}(C) \cos (2 k+1) \omega_{0} t\right) \sin \omega_{0} t \sin \varphi(t) \\
& I(t) \times\left(-\cos \omega_{0} t\right) \\
&=-A \cos \omega_{0} t-B\left(J_{0}(C)+2 \sum_{k=1}^{\infty}(-1)^{k} J_{2 k}(C) \cos 2 k \omega_{0} t\right) \cos \omega_{0} t \cos \varphi(t) \\
&+2 B\left(\sum_{k=0}^{\infty}(-1)^{k} J_{2 k+1}(C) \cos (2 k+1) \omega_{0} t\right) \cos \omega_{0} t \sin \varphi(t)
\end{aligned}
$$

After passing through the low-pass filters, the output signal are a pair of non-strict orthogonal components $I_{x}(t)$ and $I_{y}(t)$

$$
\begin{aligned}
I_{x}^{\prime}(t) & =B J_{1}(C) \sin \varphi(t) \sin \Delta \varphi \\
I_{y}^{\prime}(t) & =B J_{1}(C) \sin \varphi(t) \cos \Delta \varphi
\end{aligned}
$$

After dividing Eq. (18) by Eq. (19) can obtain

$$
I_{d i v}^{\prime}(t)=\tan \Delta \varphi
$$

So $\Delta \varphi$ can be obtain by the operation of arctangent.

\section{Simulation and Analysis}

In the system of this paper, the sampling frequency $f_{s}$ of the interference signal was $10 \mathrm{MHz}$, the carrier frequency $f_{0}$ was $1 \mathrm{MHz}$, the sensing phase signal frequency $f$ was $1 \mathrm{kHz}$. The carrier phase delay took a step length of $0.001 \pi \mathrm{rad}$, rising from $-\pi$ rad to $\pi \mathrm{rad}$. As shown in Fig. 3, the carrier phase delay can influence the amplitude of the demodulation signal. The precise amplitude can not be obtained because of the existence of the carrier phase delay. Especially, when the carrier phase delay $\Delta \varphi= \pm \pi / 2, \cos \Delta \varphi$ is equal to 0 . It leads to the demodulation failure. 


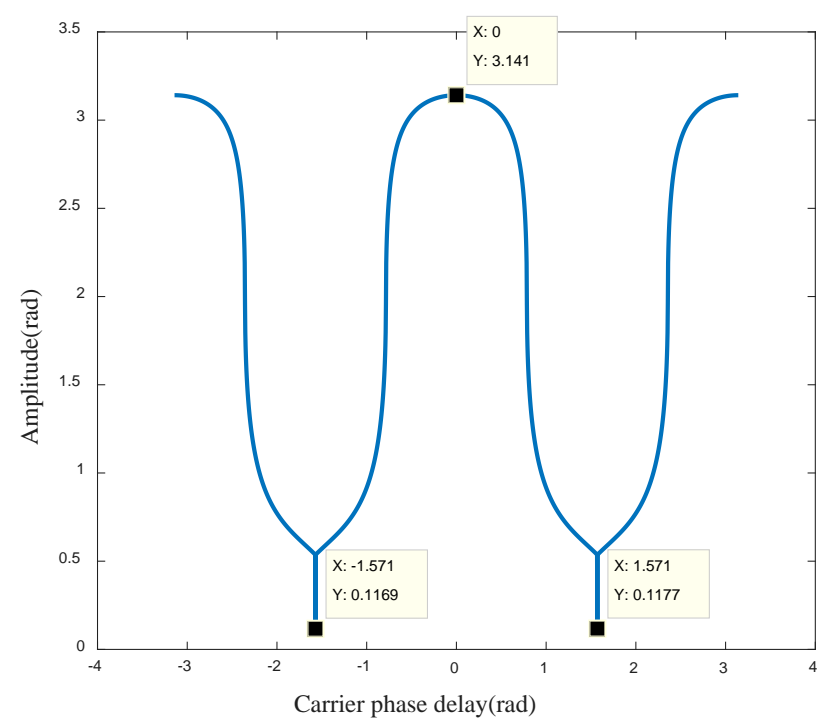

Fig. 3. The dependence of amplitude on carrier phase delay

Fig. 4(a) reflects the influence of the carrier phase delay on the demodulation performance. The carrier phase delay causes the decrease of SINAD. As shown in Fig. 4(b), the waveform of demodulation results have serious harmonic distortion when $\Delta \varphi$ are $0.1 \pi \mathrm{rad}, 0.2 \pi \mathrm{rad}$, $0.3 \pi \mathrm{rad}$ and $0.4 \pi \mathrm{rad}$, respectively. So it is essential to calculate the carrier phase delay and adjust it in the demodulation process.
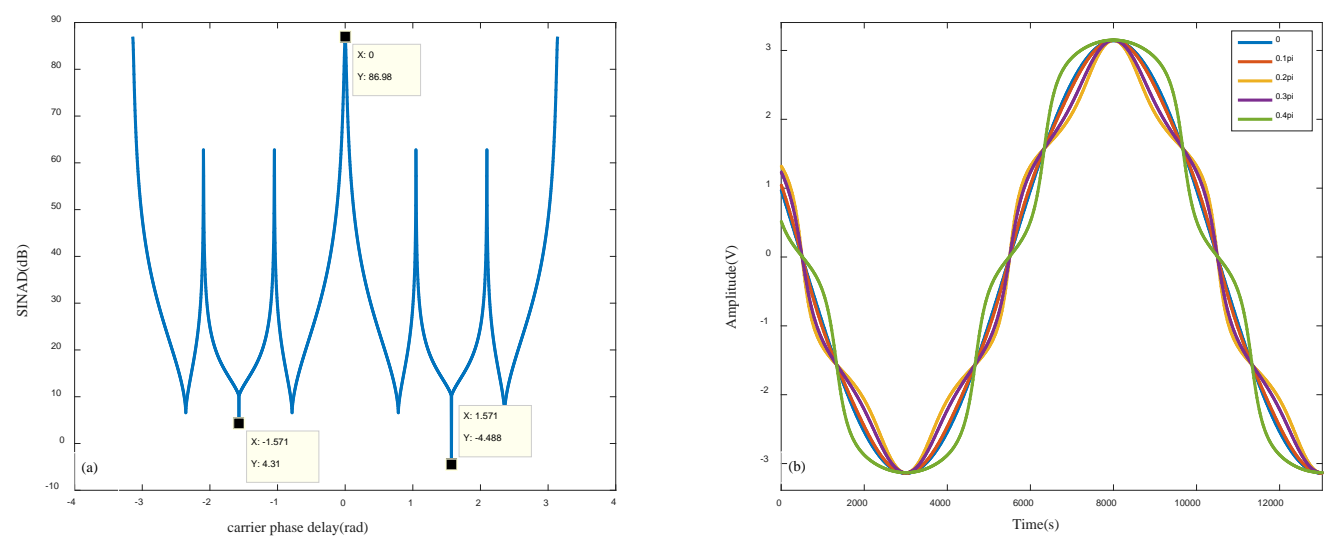

Fig. 4. The effect of carrier phase delay (a) The dependence of SINAD on carrier phase delay (b) The dependence of amplitude on carrier phase delay

As shown in Fig. 5, the carrier phase delay can be obtained by CPDS module. Then the initial phase of the carrier signal and its second harmonics can be adjusted to synchronize the carrier to depress the harmonic distortion. 


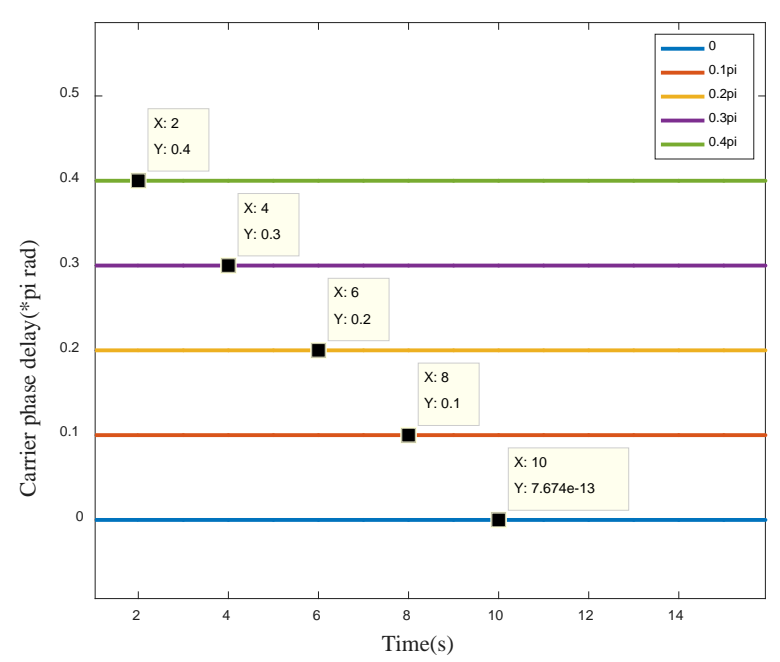

Fig. 5. The results of CPDS

\section{Experimental Results and Discussion}

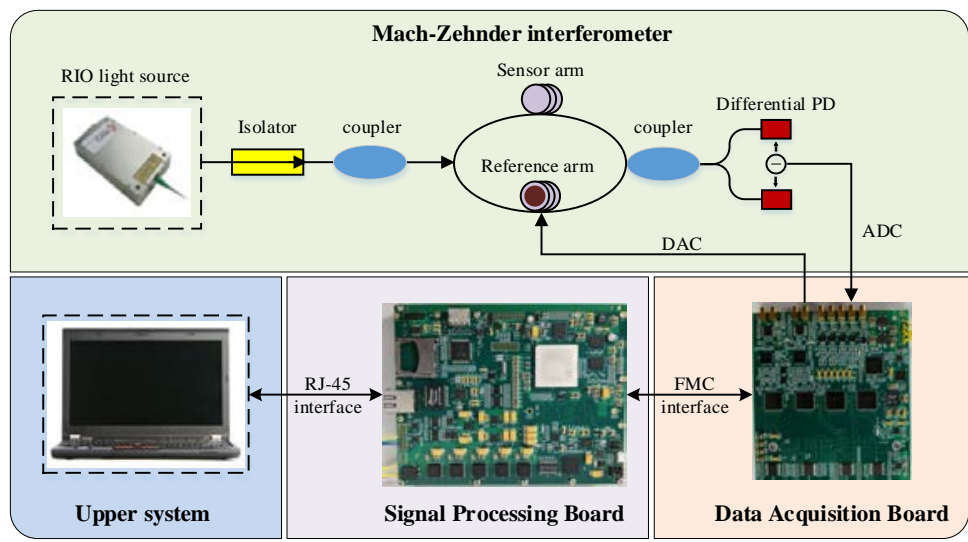

Fig. 6. Experimental setup

As shown in the Fig. 6, the practicability of CPDS algorithm is verified by the experimental. In this paper, PGC digital demodulation system is constructed on Xilinx Zynq-7100. The carrier signal generated by digital to analog converter (DAC). And the interference signal is modulated by the carrier signal through PZT modulator. The interferometric signal is emitted by Mach-Zehnder interferometer. Then the interferometric signal is sampled by ADC which is located on the data acquisition board. Then the sampled signal is demodulated on the signal processing board. The sensing phase signal can be gotten from the digital sampled signal. The demodulation system can be controlled by the upper system. 


\subsection{Carrier phase delay synchronization module}

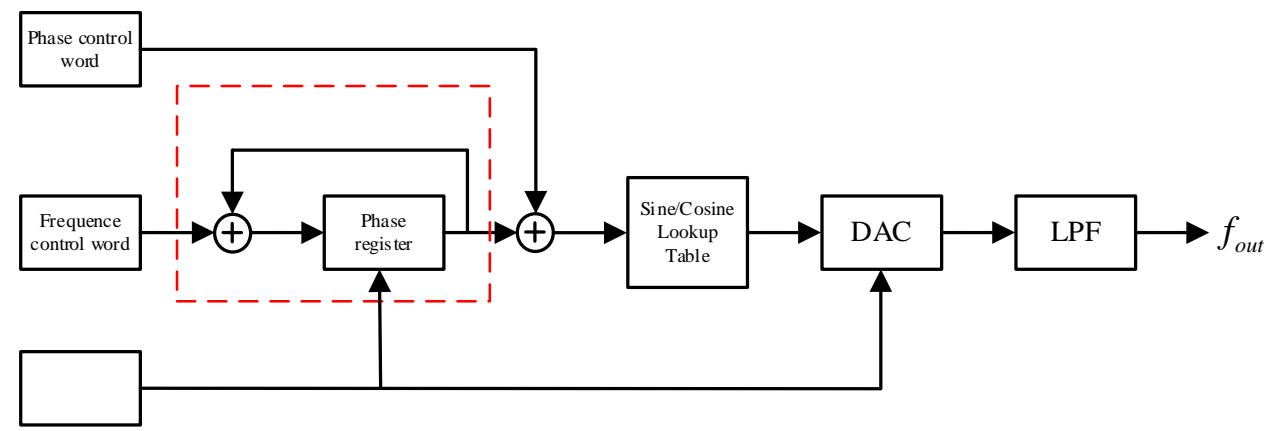

Fig. 7. The principle of direct digital synthesizers

As shown in Fig. 7, DDS are vital devices in a lot of digital processing systems, which always adopt the digital lookup table. The digital lookup table is composed by a real or complex valued sinusoid. The phase accumulator is composed of adder and phase register, which accumulates the frequency control word once a system clock. The phase accumulator outputs the phase of the sinusoidal signal. The phase is used as the sampling address of the lookup table which stores sampling values of a sinusoidal signal. In this way, the waveform sampling value can be obtained by looking up the lookup table. Then the phase-to-amplitude conversion can be completed.

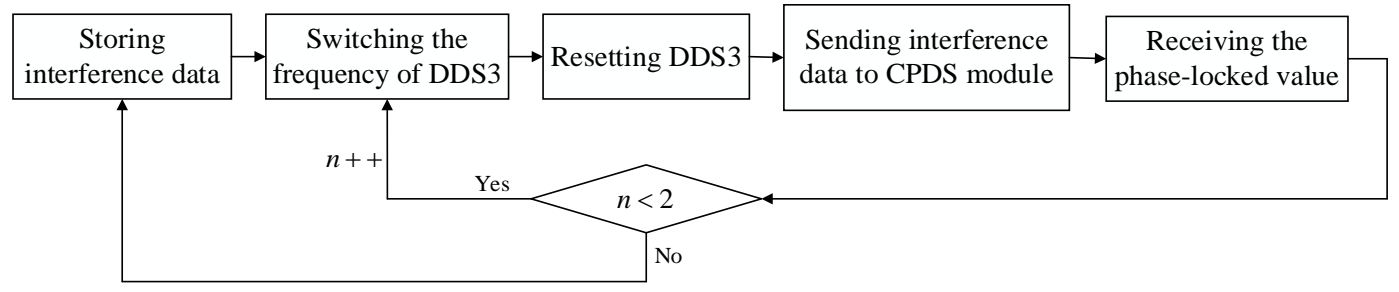

Fig. 8. Flow diagram of CPDS module

The flow diagram of CPDS module is shown in Fig. 8, Firstly, a section of interference data is stored. Then, the frequency of a DDS2 is set to $1 \mathrm{MHz}$. At the moment, $n$ is equal to 1 . Next, the DDS2 is reset. After that, the interference data is sent to CPDS module. The carrier phase delay value $\Delta \varphi$ can be obtained by orthogonal phase-locked module. Then the phase-locked value is received and converted into the phase control word of DDS1 to adjust the initial phase of the fundamental carrier signal. Finally, $n$ value is judged whether less than 2 or not. When $n$ is equal to 2, the frequency of a DDS2 is set to $2 \mathrm{MHz}$. The above operations are repeated to obtained the phase delay of the second-harmonic carrier signal.

\subsection{Experimental verification for CPDS}

In order to verify the practicability of CPDS in eliminating the harmonic distortion, the demodulation results without CPDS and the demodulation results with CPDS are tested, respectively. On the hardware platform, the sampling frequency $f_{s}$ is $10 \mathrm{MHz}$, the carrier frequency $f_{0}$ is $1 \mathrm{MHz}$, the sensing phase signal frequency $f$ is $1 \mathrm{kHz}$. As shown in Fig. 9, the SINAD of the demodulated phase signal without CPDS is $25.22 \mathrm{~dB}$. As shown in Fig. 10, the SINAD of the demodulated phase signal with CPDS is 81.21dB. Comparing Fig. 9 and Fig. 10, 
it can be seen that the system without CPDS has obvious harmonic distortion. Therefore, the carrier phase delay brings serious harmonic distortion to demodulation system. So it is necessary to use CPDS to suppress the nonlinear distortion.

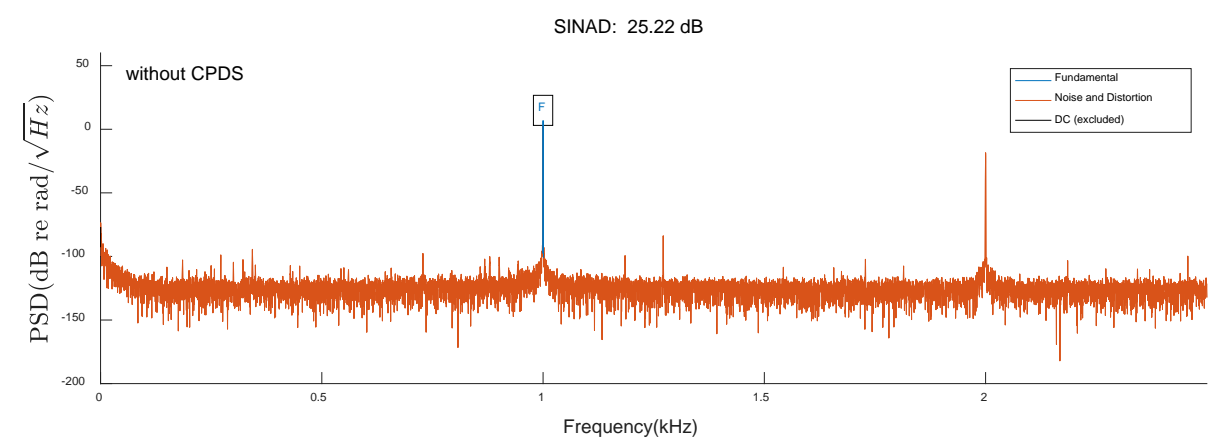

Fig. 9. SINAD analysis of the demodulated phase signal without CPDS

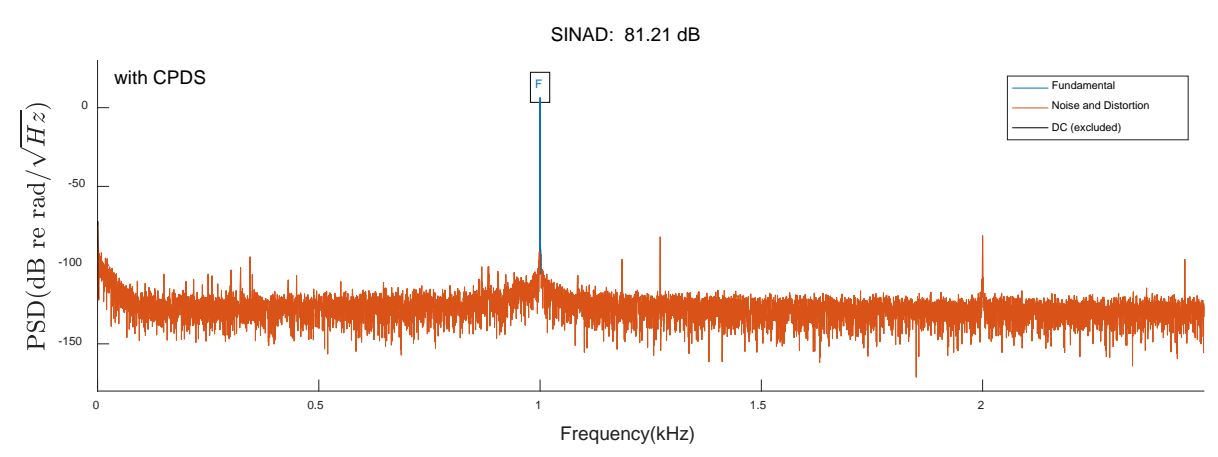

Fig. 10. SINAD analysis of the demodulated phase signal with CPDS

\section{Conclusion}

In this paper, a CPDS algorithm is proposed to automatic compensate the carrier phase delay. The effect of carrier phase delay and the effectiveness of CPDS algorithm are verified in theory. And the theories are verified by simulation further. The improved PGC-Arctan demodulation system based on CPDS algorithm is implemented on SOC. The results of the experiments show that PGC-Arctan with CPDS can effectively decrease the harmonic distortion which is caused by the carrier phase delay. The demodulation results of the hardware system are improved significantly. Compared to the conventional PGC-Arctan demodulation system, the improved PGC-Arctan demodulation system has a much better SINAD.

\section{Acknowledgements}

This work is supported by the Natural Science Foundation of Heilongjiang Province (JJ2019LH2398), the Fundamental Research Funds for the Central Universities (3072020CFT0801), Key Laboratory of Advanced Marine Communication and Information Technology, Ministry of Industry and Information Technology, Harbin Engineering University, Harbin, China. 


\section{References}

[1] P. F. Wang, G. Brambilla, M. Ding, Y. Semenova, Q. Wu and G. Farrell, "High-sensitivity, evanescent field refractometric sensor based on a tapered, multimode fiber interference," Optics Letters, vol. 36, no. 12, pp. 2233-2235, June, 2011. Article (CrossRef Link)

[2] T. G. Giallorenzi, J. A. Bucaro, A. Dandridge, G. H. Sigel, J. H. Cole, S. C. Rashleigh and R. G. Priest, “Optical Fiber Sensor Technology," IEEE Transactions on Microwave Theory and Techniques, vol. 30, no. 4, pp. 472-511, April, 1982. Article (CrossRef Link)

[3] J. D. Chen, T. Y. Chang, Y. Yang, W. Z. Gao, Z. M. Wang and H. L. Cui, "Ultra-Low-Frequency Tri-Component Fiber Optic Interferometric Accelerometer,” IEEE Sensors Journal, vol. 18, no. 20, pp. 8367-8374, October, 2018. Article (CrossRef Link)

[4] B. Culshaw and A. Kersey, "Fiber-optic sensing: A historical perspective," Journal of Lightwave Technology, vol. 26, no. 9, pp. 1064-1078, May, 2008. Article (CrossRef Link)

[5] S. Y. Chen, Y. Zhang, Z. W. He and J. B. Nie, "A novel attention cooperative framework for automatic modulation recognition,” IEEE Access, vol. 8, pp. 15673-15686, January, 2020. Article (CrossRef Link)

[6] J. Nie, Y. Zhang, Z. He, S. Chen, S. Gong and W. Zhang, "Deep Hierarchical Network for Automatic Modulation Classification,” IEEE Access, vol. 7, pp. 94604-94613, July, 2019.

Article (CrossRef Link)

[7] J. D. Chen, W. Z. Gao, T. Y. Chang, W. Sun, Z. M Wang, M. Yu, Y. Yang, Q. J. Fu and H. L. Cui, "Method for Determining the Resonance Frequency of a Fiber Optic Interferometric Accelerometer," IEEE Transactions on Instrumentation and Measurement, vol. 67, no. 3, pp. 698-705, March, 2018. Article (CrossRef Link)

[8] J. Han, W. T. Zhang, D. S. Jiang, W. G. Zhang and F. Li, "Fiber optic 3-component seismometer," Photonic Sensors, vol. 4, pp. 102-107, January, 2014. Article (CrossRef Link)

[9] B. Wu, Y. Yuan, J. Yang, A. Zhou and L. Yuan, "Improved Signal Demodulation Method in Optical Fiber Seismometer,” Sensor Letters, vol. 10, no. 7, pp. 1400-1404(5), July, 2012. Article (CrossRef Link)

[10] Q. P. Shi, T. Qian, L. W. Wang, C. D. Tian, H. Y. Zhang, M. Zhang, Y. B. Liao, H. H. Wang, X. Zeng and L. J. Huang, "Performance improvement of phase-generated carrier method by eliminating laser-intensity modulation for optical seismometer,” Optical Engineering, vol. 49, no. 2, February, 2010. Article (CrossRef Link)

[11] J. D. Chen, T. Y. Chang, Q. J. Fu, J. P. Lang, W. Z. Gao, Z. M. Wang, M. Yu, Y. B. Zhang and H. L. Cui, "A Fiber-Optic Interferometric Tri-Component Geophone for Ocean Floor Seismic Monitoring,” Sensors, vol. 17, no. 1, January, 2017. Article (CrossRef Link)

[12] G. Q. Wang, T. W. Xu and F. Li, "PGC Demodulation Technique with High Stability and Low Harmonic Distortion,” IEEE Photonics Technology Letters, vol. 24, no. 23, pp. 2093-2096, December, 2012. Article (CrossRef Link)

[13] A. Dandridge, A. Tveten and T. Giallorenzi, "Homodyne Demodulation Scheme for Fiber Optic Sensors Using Phase Generated Carrier,” IEEE Journal of Quantum Electronics, vol. 18, no. 10, pp. 1647-1653, October, 1982. Article (CrossRef Link)

[14] S. C. Huang, Y. F. Huang and Z. Z. Wu, "Sensitivity normalization technique of PGC demodulation with low harmonic distortion and high stability using laser modulation to generate carrier signal,” Sensors and Actuators A-Physical, vol. 174, no. 1, pp. 198-206, February, 2012. Article (CrossRef Link)

[15] T. R. Christian, P. A. Frank and B. H. Houston, "Real-time analog and digital demodulator for interferometric fiber optic sensors,” in Proc. of SPIE 2191, Smart Structures and Materials 1994: Smart Sensing, Processing, and Instrumentation, 1994. Article (CrossRef Link)

[16] H. Wang, L. L. Guo, Z. Dou and Y. Lin, “A new method of cognitive signal recognition based on hybrid information entropy and D-S evidence theory,” Mobile Networks and Applications, vol. 23, no. 4, pp. 677-685, August, 2018. Article (CrossRef Link) 
[17] C. Z. Shi, Z. Dou, Y. Lin and W. W. Li, "Dynamic threshold-setting for RF-powered cognitive radio networks in non-Gaussian noise,” Physical Communication, vol. 27, no. 4, pp. 99-105, February, 2018. Article (CrossRef Link)

[18] C. M. Zhou, Y. D. Pang, Q. Li, X. Chen, Q. N. Xu, C. G. Zhao, H. R. Zhang, Z. W. Tu, J. B. Huang, H. C. Gu and D. Fan, "Demodulation of a Hydroacoustic Sensor Array of Fiber Interferometers Based on Ultra-weak Fiber Bragg Grating Reflectors Using a Self-referencing Signal,” Journal of Lightwave Technology, vol. 37, no. 11, pp. 2568-2576, June, 2019. Article (CrossRef Link)

[19] A. N. Nikitenko, M. Y. Plotnikov, A. V. Volkov, M. V. Mekhrengin and A. Y. Kireenkov, "PGC-Atan Demodulation Scheme with the Carrier Phase Delay Compensation for Fiber-Optic Interferometric Sensors,” IEEE Sensors Journal, vol. 18, No. 5, pp. 1985-1992, January, 2018. Article (CrossRef Link)

[20] Y. Liu, L. W. Wang, C. D. Tian, M. Zhang and Y. B. Liao, “Analysis and Optimization of the PGC Method in All Digital Demodulation Systems,” Journal of Lightwave Technology, vol. 26, no. 17, pp. 3225-3233, September, 2008. Article (CrossRef Link)

[21] S. H. Zhang, L. P. Yan, B. Y. Chen, Z. Y. Xu and J. D. Xie, "Real-time phase delay compensation of PGC demodulation in sinusoidal phase-modulation interferometer for nanometer displacement measurement," Optics Express, vol. 25, no. 1, pp. 472-485, January, 2017. Article (CrossRef Link)

[22] S. C. Huang and H. Lin, "Modified phase-generated carrier demodulation compensated for the propagation delay of the fiber,” Applied Optics, vol. 46, no. 31, pp. 7594-7603, July, 2007. Article (CrossRef Link)

[23] S. W. Li, S. Y. Shao, H. P. Mei, Q. L. Hao and R. Z. Rao, “Analysis and mitigation of the carrier phase delay effect of the digital phase generated carrier algorithm,” Applied Optics, vol. 56, no. 3, pp. 731-738, January, 2017. Article (CrossRef Link)

[24] J. D. Xie, L. P. Yan, B. Chen and Y. T. Lou, "Extraction of Carrier Phase Delay for Nonlinear Errors Compensation of PGC Demodulation in an SPM Interferometer," Journal of Lightwave Technology, vol. 37, no. 13, pp. 3422-3430, May, 2019. Article (CrossRef Link)

[25] T. Y. Chang, J. P. Lang, W. Sun, J. D. Chen, M. Yu, W. Z. Gao and H. L. Cui, "Phase Compensation Scheme for Fiber-Optic Interferometric Vibration Demodulation," IEEE Sensors Journal, vol. 17, no. 22, pp. 7448-7454, September, 2017. Article (CrossRef Link)

[26] Y. Li, Z. B. Liu, Y. Liu, L. Ma, Z. W. Tian and S. S. Jian, "Interferometric vibration sensor using phase-generated carrier method,” Applied Optics, vol. 52, no. 25, pp. 6359-6363, October, 2018. Article (CrossRef Link)

[27] Z. H. Yu, Q. Zhang, M. Y. Zhang, H. L. Dai, J. J. Zhang, L. Liu, L. J. Zhang, X. Jin, G. F. Wang and G. Qi, "Distributed optical fiber vibration sensing using phase-generated carrier demodulation algorithm,” Applied Physics B-Lasers and Optics, vol. 124, May, 2018. Article (CrossRef Link)

[28] X. H. Wang, S. C. Piao, J. S. Fu and X. M. Li, "Automatic carrier signal track algorithm in all-digital PGC demodulation scheme for optical interferometric sensors," Journal of Optical Technology, vol. 84, no. 4, pp. 265-269, April, 2017. Article (CrossRef Link) 


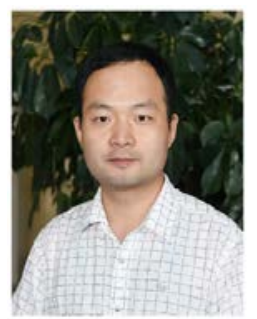

Changbo Hou received the B.S and M.S degree in College of Information and Communication Engineering from Harbin Engineering University, Heilongjiang, China, in 2008 and 2011, respectively. He is currently a Lecturer with the College of Information and Communication Engineering, Harbin Engineering University, where he is also a Doctor in the Key Laboratory of In-fiber Integrated Optics, Ministry Education of China. His research interests include wideband signal processing, optical sensors, image processing and deep learning.

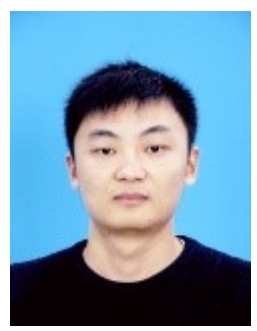

Shuai Guo received the B.S. and M.S degree in College of Information and Communication Engineering from Harbin Engineering University, Heilongjiang, China, in 2017 and 2020, respectively. His research interests include signal processing, parameter estimation, and the demodulation algorithms of optical-fiber sensors. 